\title{
Basement Membrane: Carbohydrates and X-Ray Diffraction ${ }^{1}$
}

\author{
P. A. Lee, ${ }^{2}$ K. Blasey, I. J. Goldstein, and G. B. Pierce ${ }^{3}$ \\ Departments of Pathology and Biochemistry, The University of Michigan School of Medicine, \\ Ann Arbor, Michigan 48104
}

Received March 10, 1969

The demonstration that epithelial cells of mouse and rat synthesize basement membrane antigens in tissue culture has established the concept that epithelia in general synthesize basement membrane (Pierce et al., 1964; Pierce and Nakane, 1967). Epithelial basement membranes contain hydroxyproline (Mukerjee et al., 1965). In addition, these membranes contain antigens shared by collagen and other antigens distinct from those of the connective tissue. This raises important questions regarding the nature of basement membrane: Is it collagen, a mucoprotein to be distinguished from collagen, or a combination of collagen and a mucoprotein?

A major obstacle in determination of the chemical composition of basement membrane has been the difficulty in obtaining it free from connective tissue contamination. Even carefully prepared glomeruli may be heavily contaminated by periglomerular fibrous tissue or by connective tissue elements at the point of entry and exit of arterioles. Thus, the presence of collagenous components in glomerular basement membrane, as determined by chemical analysis (Spiro, 1967; Kefalides and Winzler 1966; Kefalides 1968) and x-ray diffraction, may represent a contaminant and must be confirmed on material free of connective tissue.

A parietal yolk sac carcinoma of the mouse has been developed which synthesizes epithelial hasement membrane in vitro or in the ascites, situations free of connective tissue cells and their products. In a previous study of the chemical composition of this neoplastic basement membrane (Mukerjee et al., 1965) hydroxyproline and hydroxylysine were found in amounts comparable to those in collagen and reticulin, but glycine was present in about half the amount characteristic of collagen. It contained more neutral sugar and hexosamine than is characteristic of collagen, and a pentose believed, on the basis of chromatographic evidence, to be lyxose. Because of the limitations of the method employed for the identification of lyxose and because of the potential importance of this observation, a detailed study of the carbohydrate portion of this basement membrane was undertaken. In addition, high-angle $\mathrm{x}$-ray diffrac-

\footnotetext{
This work was supported in part by grants E-105 I from the American Cancer Society and CA-08201 from the U.S.P.H.S.

Medical Scientist Fellow of the Life Insurance Medical Research Fund.

American Cancer Society, Professor of Pathology. Present Address: Department of Pathology, University of Colorado Medical Center, Denver, Colarado.
} 
tion patterns were obtained from the isolated basement membrane in order to determine whether or not collagen or a collagen-like structure was present in this basement membrane.

\section{MATERIAIS AND METHODS}

Epithelial basement membrane (EBM) was prepared as previously described (Pierce et al., 1964), from the ascites form of the parietal yolk sac carcinoma.

Samples for monosaccharide analyses were hydrolyzed in $1.0 \mathrm{~N} \mathrm{H}_{2} \mathrm{SO}_{4}$ for 8 hours at $100^{\circ} \mathrm{C}$ in sealed tubes. The phenol-sulfuric acid method (DuBois et al., 1956) was employed to estimate the total neutral sugar fraction. Neutral sugars were isolated by the method of Spiro (1960) and chromatographed or measured colorimetrically. The solvent systems employed in descending paper chromatography on Whatman No. 1 paper were: A, ethyl acetate-pyridinewater $(8: 2: 1 \mathrm{v} / \mathrm{v})$, (Mukerjee, et al., 1965), B, (10:4:3 v/v) (Whistler and Hickson, 1955), and C, n-butanol-water-azotrope (Partridge and Westfall, 1948). Glucose and galactose concentrations were determined enzymatically utilizing kits purchased from Worthington Biochemical Corporation. Mannose was isolated chromatographically and quantitated by the phenol-sulfuric acid colorimetric technique (DuBois et al., 1956). Fucose was estimated by the method of Dische and Shettles (1948) by absorption at $490 \mathrm{~m} \mu$. Pentose was isolated by chromatography using solvent $\mathrm{D}$ ethyl acetate-pyridine- $n$-butanolbutyric acid-water (10:10:5:1:5 v/v) (Mukerjee and Sri Ram, 1964), and quantitated by the method developed by Boas (1953).

Uronic acid was assayed by the method of Dische (1947). Sialic acid measurements were conducted by the thiobarbituric acid assay (Warren, 1959), after heating at $80^{\circ} \mathrm{C}$ for 1 hour in $0.1 \mathrm{~N}_{2} \mathrm{SO}_{4}$ and passing the hydrolyzate through a Dowex $2-\mathrm{X}^{8}$ column according to Svennerholm (1958). Deoxyribonucleic acid and ribonucleic acid were isolated from the EBM preparation using the method of Scott et al. (1956) and estimated spectrophotometrically. Total nitrogen content of EBM was determined by the micro-Kjeldahl method.

Samples of EBM, and collagen from tendons of the tails of mice, prepared in a similar manner, were used for the study employing $x$-ray diffraction. These samples were placed in quartz capillaries and high-angle $x$-ray diffraction patterns were obtained using a flat-plate camera, Ni-filtered, CuK radiation, exposure times of 10-20 hours, and specimen-to-film distances of $6 \mathrm{~cm}$.

\section{RESULTS}

Using the techniques of partition paper chromatography and specific enzymatic assays, the monosaccharides present in EBM were identified as D-galactose, D-glucose, mannose, fucose, deoxyribose, and ribose. The amounts are listed in Table I. The total content of monosaccharide in hydrolyzed EBM was $7.25 \%$ as determined by the sum of the amounts of the individual sugars.

DNA and RNA have been found in the basement membrane and are presumably the source of the deoxyribose and ribose. When EBM was digested with $1 \mathrm{~N} \mathrm{NaOH}$, neutralized with $0.5 \mathrm{~N} \mathrm{HCl}$, and incubated with deoxyribonuclease or ribonuclease, the degree of absorption at $260 \mathrm{~m}_{\mu}$ (the wavelength of 
TABLE I

Carbohydrate Composition of Epithelial Basement Membrane

\begin{tabular}{|c|c|c|c|c|}
\hline & $\mathrm{Gm} / 100 \mathrm{gm}$ dry weight & $\begin{array}{c}\text { Moles monosac- } \\
\text { charide/Mole } \\
\text { nitrogen }\end{array}$ & $\begin{array}{l}\text { Molar ratio to } \\
\text { hydroxyproline }^{a}\end{array}$ & $\begin{array}{c}\text { Number of } \\
\text { analyses }\end{array}$ \\
\hline Galactose & $1.32 \pm 0.09$ & 14.5 & .145 & 4 \\
\hline Glucose & $1.98 \pm 0.02$ & 22.0 & .217 & 5 \\
\hline Mannose & $2.01 \pm 0.04$ & 21.5 & .220 & 5 \\
\hline Fucose & $1.10 \pm 0.06$ & 13.2 & .132 & 4 \\
\hline Pentose & .85 & 11.4 & .112 & 2 \\
\hline Glucosamine & $2.02 \pm 0.04$ & 19.8 & .165 & 5 \\
\hline Sialic acid & .22 & 1.6 & .016 & 3 \\
\hline
\end{tabular}

${ }^{a}$ Calculated using hydroxyproline values previously reported for EBM (Mukerjee et al., 1965).

maximum absorption of nucleic acid bases) was markedly reduced. These nucleic acids probably reflect the degree of cellular contamination of the basement membrane material.

Sufficient separation of mannose $\left({ }^{R}\right.$ galactose $\left.{ }^{1.72}\right)$ from glucose $\left({ }^{R}\right.$ galactose $\mathrm{e}^{1.27}$ ) and galactose was obtained after 20 hours development with solvent A for quantitative recovery. Epithelial basement membrane contained 2.0\% mannose and 21.5 moles of mannose per mole of nitrogen. Fucose constituted $1.1 \%$ of EBM. Lyxose was not observed in any of the preparations of EBM tested.

The content of glucosamine in EBM was $2.0 \%$. There were 19.8 moles of glucosamine for each mole of nitrogen. Neither galactosamine nor mannosamine were found by chromatographic methods. Sialic acid comprised only $0.22 \%$ of dry EBM or 1.56 moles of sialic acid per mole of nitrogen. No uronic acid was found.

The high-angle $x$-ray diffraction patterns obtained from freeze-dried collagen and epithelial basement membrane are shown in Figs 1 and 2, respectively. The collagen powder pattern contained the following intensity maxima: (1) a sharp, intense maximum corresponding to a Bragg spacing of $11.1 \AA$; (2) a moderately sharp, but weak maximum corresponding to a Bragg spacing of $7.0 \AA$; (3) a broad, intense maximum whose peak position corresponds to a Bragg spacing of $4.5 \AA$; and (4) a sharp, moderately intense maximum corresponding to a Bragg spacing of $2.9 \AA$.

The epithelial basement membrane powder pattern contained the following intensity maxima: (1) a moderately sharp, intense maximum corresponding to a Bragg spacing of $10.5-11.0 \AA$ (no well-defined peak position: Its integrated intensity classified it as relatively intense); (2) a moderately sharp, but very weak maximum corresponding to a Bragg spacing of $7.0 \AA$; (3) a broad, intense maximum whose peak position corresponds to a Bragg spacing of $4.46 \AA$; (4) a sharp, moderately intense maximum corresponding to a Bragg spacing of $2.9 \AA$; and (5) a sharp, moderately intense maximum and a sharp, weak maximum corresponding to Bragg spacings of 3.35 and $3.14 \AA$, respectively. 

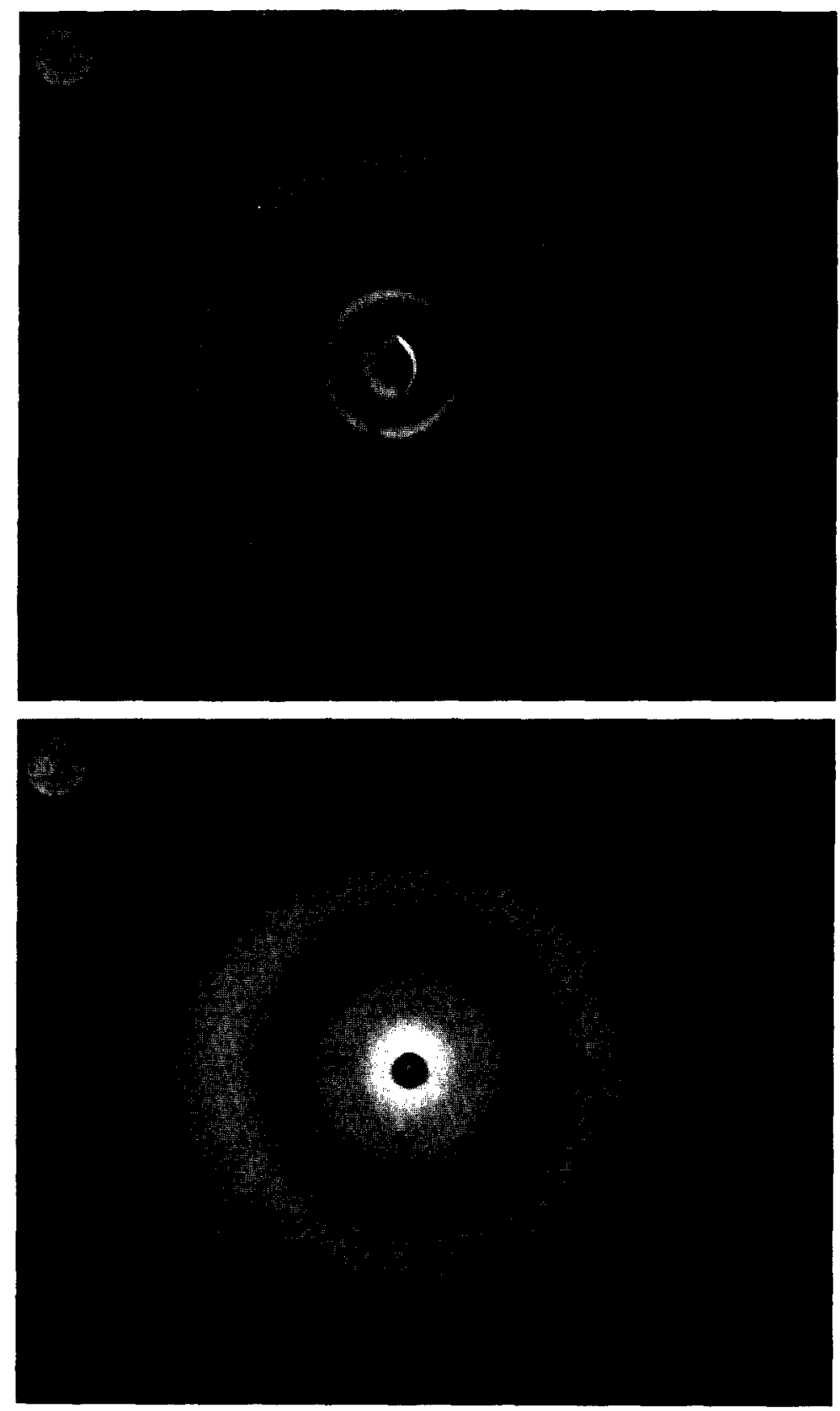

Fig. 1. High-angle $\mathrm{x}$-ray diffraction pattern of freeze-dried collagen. Intensity maxima corresponding to Bragg spacings of $11.1 \AA(1), 7.0 \AA, 4.5 \AA(2)$, and $2.9 \AA$ (3) are present.

Fig. 2. Epithelial basement membrane powder $x$-ray diffraction pattern. Note intense maximum corresponding to Bragg spacings of $10.5 \AA$ (1), 7.0 $\AA, 4.46 \AA$ (2), $3.35 \AA$ (3), $3.14 \AA$ (4), and $2.9 \AA$ (5). 


\section{DISCUSSION}

The parietal yolk sac carcinoma employed in the present study is one of three carcinomas of the mouse which has been shown capable of synthesizing basement membranes in vitro, in the absence of connective tissue cells or fibers. These tumors secreted the same basement membrane antigens, one of which was common to all basement membranes associated with normal epithelia of the mouse. Consequently, this neoplastic basement membrane, free as it is of connective tissue contamination, is a valuable substance for studying the molecular nature of basement membrane and its relationship to collagen.

In a previous study of the chemical composition of neoplastic epithelial basement membrane, Mukerjee et al. (1965) reported the presence of glucose, galactose, mannose, lyxose, glucosamine, and a trace of fucose. Using chromatographic procedures with three solvent systems, we have been unable to confirm the presence of lyxose; the pentoses present are ribose and deoxyribose. These sugars are probably derived from nucleic acids present in the material, presumably the result of contamination by cellular detritus that has escaped the sonication and washing of the preparatory procedures. In this regard, we have been unable to identify cellular organelles in the preparations by electron microscopy. Misra and Berman (1966) have reported the presence of ribonucleic acid in their preparations of human glomerular basement membrane. They believe it to be an integral part of the membrane. Spiro (1967) found small amounts of DNA and RNA and does not consider them to be a part of the glycoprotein.

D-glucose, D-galactose, mannose, fucose, glucosamine, and sialic acid have been identified in EBM hydrolyzates. These are the same monosaccharide components found by Kefalides and Winzler (1966) using canine glomerular basement membrane; Spiro (1967) using bovine glomerular basement membrane; and Misra and Berman (1966), and Lange and Markowitz (1965) using human glomerular basement membrane. With the exception of sialic acid, Lazarow and Speidel (1964) reported similar components in human GBM after digestion with $.05 \mathrm{~N} \mathrm{NaOH}$. Spiro (1967) and Lange and Markowitz (1965) found both glucosamine and galactosamine, but in confirmation of Kefalides and Winzler (1966) and Misra and Berman (1966), we found only glucosamine.

Spiro (1967) reported a total of $9.42 \%$ carbohydrate and $6.29 \%$ hexose. Kefalides and Winzler (1966) found $9.76 \%$ carbohydrate and $5.52 \%$ hexose. EBM contains a total of $9.50 \%$ carbohydrate and $5.31 \%$ hexose.

With the exception of sialic acid, the molar ratios of carbohydrate components to hydroxyproline for EBM (Table I) fall within the range of analyses of glomerular basement membrane (Spiro, 1967; Kefalides and Winzler, 1966; Misra and Berman, 1966). The small amount of sialic acid in EBM in relationship to glomerular basement membrane, may represent an intrinsic difference in the chemical composition of epithelial and endothelial basement membrane. Glomerular basement membrane contains EBM and endothelial basement 
membrane antigens; the latter may be rich in sialic acid. Similarly, the galactosamine reported in glomerular basement membrane by Lange and Markowitz. (1965) and Spiro (1967) probably represents another variation in chemical composition between EBM and glomerular basement membrane. Collagen contains less sialic acid and less hexosamine (Kefalides and Winzler 1966) than either EBM or glomerular basement membrane.

The salient features of the $\mathrm{x}$-ray diffraction studies are the intensity maxima of the collagen powder pattern corresponding to Bragg spacings of 10,4.5, and $2.86 \AA$. The first of these corresponds to the inter-coiled-coil separation of Rich and Crick's (1961) coiled-coil model for collagen. The position of the 10- $\AA$ intensity maximum is sensitive to the moisture content of the specimen and its shape is sensitive to the size of the coherent scattering unit in the plane normal to the coiled-coil axis. Hence, this maximum is very dependent on the environment and preparation of the specimen. The second intensity maximum is a composite of several reflections from the collagen structure. The third intensity maximum is most unique to the structure of collagen in that it represents a $2.86 \AA$ translational symmetry along the helical screw axis of the coiledcoil collagen structure. The coiled-coil collagen structure has 10 units in three turns with a translation along the helical screw axis of $2.86 \AA$ per unit. This reflection is sensitive to stretching and so varies from 2.86 to $2.91 \AA$.

In view of the above considerations, comparison of the high-angle $x$-ray diffraction powder patterns for collagen and EBM show remarkable agreement. It is not surprising that the 10.5-11.0 $\AA$ intensity maximum for the EBM is more broad than that of collagen. Microscopic examination of the specimens showed that the "particles" composing the EBM "powder" were considerably smaller than those of the collagen "powder." Thus, considerably greater disruption of the EBM structure occurred during specimen preparation than it did for the collagen specimen which may well have reduced the size of the coherent scattering unit for the EBM. The 4.5- $\AA$ intensity maxima are nearly identical in shape for the two specimens. Inasmuch as this intensity maximum is a composite of several reflections for the case of collagen, comparison of the shape of this maximum for the two specimens is perhaps most significant. The close agreement between the two specimens for the 2.9- $\AA$ intensity maximum is of most importance since it would indicate that EBM may well contain a coiled-coil structure with an identical translational symmetry for its helical screw axis.

Thus, it would appear that a collagen or collagen-like coiled structure is likely to be a basic structural element in EBM. This observation is compatible with our previous observation that hydroxyproline is found as an integral part of EBM (Mukerjee et al., 1965). The presence of two other intensity maxima in the diffraction pattern from EBM indicates the presence of other components in the specimen, presumably the epithelial specific mucoprotein antigen not found in collagen (Pierce and Nakane 1967).

Although the molecular nature of EBM is not known at this time, an analogy to bone may be useful. Bone is composed of collagen to which molecules of bone salts are added in well-ordered array. It would appear that basement 
membrane contains collagen to which an antigenic mucoprotein (characteristic of all epithelial basement membranes) is added. In support of this idea is Kefalides' demonstration of soluble collagen extracted from glomerular basement membrane (Kefalides 1968).

Since the epithelial origin of basement membrane has previously been shown (Pierce et al., 1964), this demonstration of collagen in epithelial basement membrane establishes unequivocally that all epithelial cells contain the genetic information for the production of collagen, and moreover, they synthesize the collagenous stroma of basement membrane.

\section{SUMMARY}

Epithelial basement membrane, synthesized by a parietal yolk sac carcinoma in vitro or in the ascites, is free of connective tissue cells and products. Carbohydrate components of this basement membrane were D-galactose, D-glucose, mannose, fucose, deoxyribuse, ribose, glucosamine, and sialic acid. Total carbohydrate content was $9.50 \%$. Galactosamine, and uronic acid were absent. This composition is compatible with previously reported analyses of basement membrane from non-neoplastic tissues.

There was similarity in four out of five intensity maxima with high-angle $x$-ray diffraction when comparing basement membrane and collagen. A collagen or collagen-like structure may therefore be a basic structural element in epithelial basement membrane.

\section{REFERENCES}

Boas, N. F. (1953). Method for the determination of hexosamines in tissues. J. Biol. Chem. 204, $553-563$.

Dische, Z. (1947). A new specific color reaction of hexuronic acids. J. Biol. Chem. 167, 189-198.

Dische, Z., and Shetrtes, L. B. (1948). Specific color reaction of methylpentoses and spectrophotometric micro-method for their determination. J. Biol. Chem. 175, 595-603.

DuBois, Michel, Gilles, K. A., Hamilton, J. K., Rebers, P. A., and Smith, Fred (1956). Colorimetric method for determination of sugars and related substance. Anal. Chem. 28, 359-356.

KefaldDEs, N. A. (1968). Isolation and characterization of the collagen from glomerular basement membrane. Biochemistry 7, 3103-3112.

Kefaldoes, N. A., and WinzLer, R. J. (1966). The chemistry of glomerular basement membrane and its relation to collagen. Biochemistry 5, 702-713.

Lange, C. F., JR., and Markowitz, A. S. (1965). Chemistry of whole glomerular basement membranes and of a soluble fraction prepared by trypsinization. Biochem. Biophys. Acta 101, 217220.

Lazarow, A., and Spridjel, C. (1964). The chemical composition of the glomerular basement membrane and its relationship to the production of diabetic complications. In "Small Blood Vessel Involvement in Diabetes Mellitus," M. D. Siperstein, A. R. Calwell, and K. Meyer, eds., pp. 127-156. Am. Inst. Biol. Sci., Washington, D. C.

Misra, R.P., and Berman, L. B. (1966). Studies on glomerular basement membrane. I. Isolation and chemical analysis of normal glomerular basement membrane. Soc. Exptl. Biol. and Med. Proc. 122, 705-710.

MukerJee, H., and SRI RAm, J. (1964). Paper chromatographic separation of glucosamine and galactosamine. Anal. Biochem. 8, 393-304.

MukerJee, H., SRi Ram, J., and Pifrce, G. B., JR. (1965). Basement membranes V. Chemical composition of neoplastic basement membrane mucoprotein. Am. J. Pathol. 46, 49-57.

PartRidge, S. M., and WestFall, R. G. (1948). Filter paper partition chromatography of sugars. Biochem. J. 42, 238-248.

Pifrce, G. B., Jr., Beais, T. F., SRI Ram, J., and Midgley, A. R., JR. (1964). Basement membranes. IV. Epithelial origin and immunological cross-reaction. Am. J. Pathol. 45, 929-962.

Pierce, G. B., JR., and NaKane, P. K. (1967). Antigens of epithelial basement membranes of mouse, rat and man: A study utilizing enzyme-labeled antibody. Lab. Invest. 17, 499-514. 
Rich, A., and CRICK, F. H. C. (1961). The molecular structure of collagen. J. Mol. Biol. 3, 483506.

Scott, J. F., Fraccastoro, A. P., and Taft, E. B. (1956). Studies in histochemistry. I. Determination of nucleic acids in microgram amounts of tissue. J. Histochem. Cytochem. 4, 1-10.

SpIRo, R. B. (1960). Studies on fetuin, a glycoprotein of fetal serum. I. Isolation, chemical composition, and physiochemical properties. J. Biol. Chem. 235, 2860-2869.

SpIRo, R. G. (1967). Studies on the renal glomerular basement membrane. Preparation and chemical composition. J. Biol. Chem. 242, 1915-1922.

Svennerholin, L. (1958). Quantitative estimation of sialic acids. Acta Chim. Scand. 12, 547-554.

Warren; L. (1959). The thiobarbituric assay of sialic acids. J. Biol. Chem. 234, 1971-1975.

WhistLeR, R. L., and Hickson, J. L. (1955). Determination of some components in corn sirups by quantitative paper chromatography. Anal. Chem. 27, 1514-1517. 